\title{
Buying Campaign Investments with Policy Concessions*
}

\author{
Greg Sasso \\ Emory University \\ gregory.sasso@emory.edu
}

\author{
Dan Alexander \\ University of Rochester \\ dan.alexander@rochester.edu
}

February 24, 2021

\begin{abstract}
We present a game-theoretic model of elections and policy implementation with special interest groups. Candidates for office choose a level of policy concessions to grant to interest groups. Interest groups then contribute to campaigns to help elect their preferred candidates. Our results offer predictions about equilibrium campaign spending, the probability a given candidate wins, and the level of policy concessions as a function of the ideological distribution of politicians and interest groups. Campaign spending increases as interest groups and candidates become more extreme relative to the median voter. In contrast, the probability a given candidate wins depends (negatively) on ideological alignment with an allied interest group. We then show that when parties can nominate candidates, they choose to nominate the most moderate candidate but choose to pair her with the most extreme interest group.
\end{abstract}

${ }^{*}$ We would like to thank Keith Schnakenberg, Benjamin Ogden, Scott Ashworth, Peter Buisseret, Gleason Judd, John Patty, Pablo Montagnes, and participants at the CSDP conference on Interest Groups, Policy Outcomes, and Representation, APSA 2018 and 2019, and SPSA 2019 for helpful comments and suggestions. 


\section{Introduction}

Interest groups may affect policy in two distinct yet strategically interrelated means. First, interest groups use campaign spending to help elect ideologically aligned politicians. Interest groups may lobby winning candidates for policy concessions. Indeed, the largest and most influential interest groups routinely engage in both campaign spending and lobbying (Tripathi et al., 2002; Lake, 2015; Crosson et al., 2020).

Existing research, however, is mixed on the links between campaign contributions and policy concessions. For example, Fouirnaies and Fowler (2021) finds no evidence that insurance companies benefit from their campaign contributions to state legislators and receive no special access. Further, Ansolabehere et al. (2003), advance the notion that contributions are not particularly effective, but may instead be a form of consumption. Fowler et al. (2020) also find contributions to have no beneficial effect for the contributing group.

On the other side, McKay (2018b) and Kalla and Broockman (2016) present systematic evidence that campaign contributions buy access, showing that politicians do not just implement policies favored by supported interest groups but also use language specifically written by groups that hosted fundraisers. Further, Fouirnaies and Hall (2018), Powell and Grimmer (2016), and McKay (2018a) show that interest groups prioritize contributions for candidates that will hold positions on relevant committees. ${ }^{1}$

We analyze a formal model of electoral competition with both policy concessions and campaign contributions to understand the strategic relationship between both tactics and to help rationalize these competing findings. Our model contains two interest group-candidate pairs (one left-leaning, one right-leaning) and an electorate. All players are policy motivated. The left-leaning interest group is more aligned ideologically with the left-leaning candidate than it is with the right-leaning candidate, with the analogous relationship holding for the right-leaning interest group-candidate pair. In the first stage, politicians commit to a level of concessions they will give the aligned interest group if they win the election. The second stage is the electoral campaign, in which interest groups may spend on the campaign of their aligned politician - spending that translates into candidate valence - and voters choose their preferred candidate. The location of the median voter is uncertain, but resolved after campaign spending. Lastly, the final policy is implemented by the winning candidate. To represent policy concessions, the policy implemented is a weighted average of the winning candidate's and interest group's ideal points, where the weight given to the interest group is the level of concessions granted by the candidate.

We explore a few examples here to preview the sorts of interactions and tradeoffs that we seek to capture

\footnotetext{
${ }^{1}$ While we focus mostly on the U.S. setting, the effects of political money are of interest worldwide. For example, Fouirnaies (2020) shows how higher campaign spending limits benefit wealthier candidates in the United Kingdom and Titl and Geys (2019), show how donations to winning politicians increases a firm's chances of receiving procurement contracts in the Czech Republic.
} 
in our model. First, consider two moderate politicians and two extreme interest groups (Figure 1, which depicts the ideal points $\hat{x}$ of the left-leaning group and politician, $G_{L}$ and $P_{L}$, and the right-leaning group and politician, $G_{R}$ and $P_{R}$, and the expected location of the median voter, $M$ ). Assume the candidates grant no concessions, so the potential final policies are simply the politicians' ideal points. Even though the interest groups have no influence on the final policy once a candidate wins office, the groups are still willing to spend some amount of money to help elect their preferred candidate. Final policy depends first and foremost on which candidate wins the election, and that induces spending from interest groups.

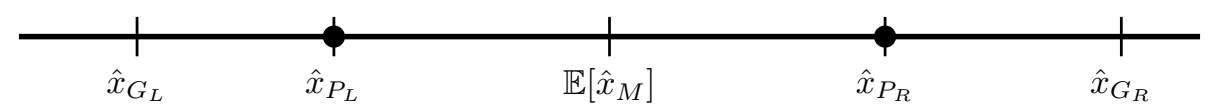

Figure 1: Interest groups relatively more extreme than their aligned politicians

Now suppose the right politician becomes even more moderate, and still assume the politicians grant no concessions (Figure 2). The right policy is now more preferred by the median voter which would put upward pressure on spending from the left interest group, but there are two other effects. The right interest group now likes the right policy less than she did before (reduced alignment), and the left interest group now likes the right policy more than she did before (reduced polarization). The net effect of the simultaneous changes in polarization and alignment is to drive down campaign spending.

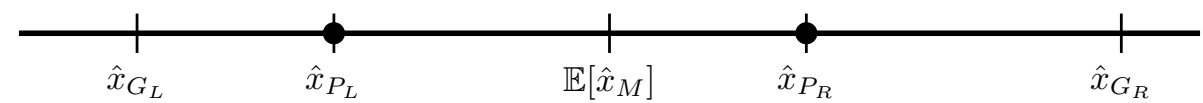

Figure 2: Relatively extreme interest groups but with a more moderate right politician than Figure 1

Finally, flip the ideal points of the left politician and left interest group and let the final policy be the left interest group's ideal point (Figure 3$).^{2}$ How would interest group behavior change? The location of the final policies have not changed even though the polarization of the politicians has changed. The voter and the right interest group's calculations thus remain the same. The left interest group, however, is now closer to both the final policy of the right politician and the final policy of the left politician. She may therefore be less willing to spend on the election because losing is not as bad as it was before, and yet may be more inclined to spend because winning is better than it was previously. Again, the net effect on spending is driven by a combination of changing both polarization and alignment at the same time.

What would be the effect of offering policy concessions to an extreme interest group? Concessions would pull the politician's implemented policy away from the politician. This more extreme policy would then be

\footnotetext{
${ }^{2}$ We will see below that final policies coincide with the ideal points of the members who are the most moderate on each side.
} 


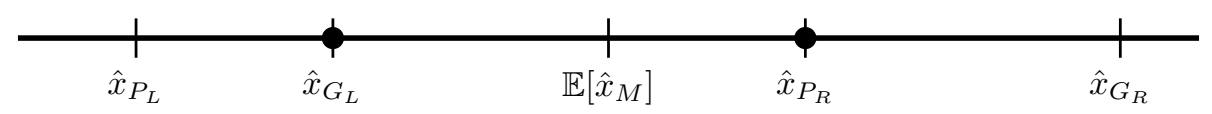

Figure 3: Moderate Left Interest Group, Extreme Right Interest Group

farther away from the median voter, making it less likely the politician would win. Both of these forces make concessions unappealing to the politician. However, concessions would also push the interest group to spend more on the campaign, making it more likely the politician would win. Offering concessions also affects the opposing interest group's behavior. Because concessions make the implemented policy more extreme, losing is now worse for the opposing interest group. Therefore, the opposing interest group also increases spending when an extreme interest group receives concessions.

Most of the effects are reversed for moderate interest groups. While the implemented policy still moves away from the politician, the direction is now towards the median voter making victory more likely. Additionally, a more moderate policy makes losing less harsh for the opposing interest group. This drives down opposing group spending. From the politician's perspective, granting concessions to the moderate groups offers more benefits than granting concessions to extreme interest groups.

These simple examples illustrate several key insights from the model. First, there is a policy benefit for an interest group from its aligned candidate winning, regardless of any concessions given. Therefore, interest groups are willing to offer contributions even when they receive no concessions. Second, a few relationships emerge as central to understanding equilibrium campaign spending and lobbying. In particular, the degree of ideological alignment within the right- and left-leaning group-politician pairs is as important as the degree of polarization between opposing politicians in determining the levels of both the concessions and the contributions. Additionally, candidate and interest group behavior rely inextricably on whether the interest group is more or less extreme than its preferred candidate. Candidates provide different levels of concessions to relatively extreme versus relatively moderate interest groups and in turn, these groups spend different amounts on the campaigns. One contribution of our analysis is to demonstrate that considering just polarization without taking into account alignment (or vice versa) would provide only a partial account of underlying mechanisms. Our study is geared towards formalizing and generalizing intuitions about the various effects of changes in polarization and alignment, individually and taken together.

Depending on the relative extremism of an interest group and its aligned politician, politicians either grant full concessions or no concessions. Specifically, politicians grant full concessions to interest groups that are more moderate than they are, such that the politician implements the group's ideal point if elected; politicians grant no concessions to interest groups that are more extreme than they are, such that the politician implements her own ideal point if elected. Crucially, this does not mean a group relatively more 
extreme than its aligned politician does not spend on that politician's campaign. In fact, it contributes more than if it were relatively more moderate because it more strongly prefers to see its aligned politician's ideal point as a policy rather than the ideal point of the opposed interest group or politician. While policy is determined by the two relatively moderate players on each side of the median, be they interest groups or politicians, spending depends on the ideological distribution of all players.

The impact of relative extremism on equilibrium concessions suggests the need to focus also on the relative position of groups and candidates, not just their alignment or individual ideology. Analyzing the model in terms of single ideal points is infeasible - we will always need to consider cases in which interest groups are more and less extreme when solving for equilibrium or performing comparative statics. To keep track of levels of polarization as well as the alignment and ordering within a candidate-group pair, we reparameterize the model in terms of these relative quantities. Specifically, we study the signed distance between the ideal points of various pairs of actors. We make no symmetry assumptions and only require same-side politicians and interest groups be more aligned with each other than with politicians or interest groups on the opposing side. Not only does this streamline the analysis, but it disentangles the effects of polarization, alignment, and relative extremism while also emphasizing the model's most empirically-relevant features and predictions.

For example, we find that increased campaign spending on a candidate's campaign does not correlate with a greater probability of that candidate winning the election. An interest group's spending increases as it becomes more extreme, but the probability that its aligned candidate wins decreases as the group and the candidate become more aligned in an absolute sense. For a group that is more moderate than its aligned candidate, becoming more extreme entails becoming more aligned with the candidate, so in this region becoming more extreme leads to greater spending but a lower probability of winning. For a group that is more extreme than its aligned candidate, becoming more extreme entails becoming less aligned with the candidate, so spending and the probability of winning both increase as the group grows more extreme.

We are also able to compare the spending of relatively moderate interest groups to relatively extreme groups. Specifically, if one interest group is more extreme than its aligned politician while the other is more moderate than its aligned politician, the relatively extreme group always spends more than the relatively moderate group on the election. The utility difference from winning rather than losing is greater for an extreme group; therefore they spend more than a moderate group on the campaign even though the extreme interest group receives no concessions. Such insights again demonstrate the need for a nuanced yet parsimonious characterization of the ideological distribution of relevant actors.

We then extend our baseline model by letting parties select a candidate before the policy concessions stage. We find that the parties will nominate the most moderate candidates they can. However, the optimal environment for the parties includes the maximally extreme aligned interest group. A maximally moderate 
politician and maximally extreme interest groups leverages the electoral benefits of moderate politicians with the spending benefits of an extreme interest group. This result also suggests one reason why parties and moderate politicians may form coalitions with extreme interest groups over groups that are more ideologically aligned.

Prior work has generally considered campaign spending or policy influence in isolation, rather than jointly. One exception, Felli and Merlo (2007), allows interest groups to both support campaigns and lobby elected politicians. However, interest groups never use both tactics in equilibrium. Our paper shows both campaign spending and influence on policy location on behalf of the same politician will occur. The empirical literature supports this approach, showing that many interest groups, including some of the biggest groups, spend money on both campaigns and lobbying for the same politicians (Tripathi et al., 2002; Lake, 2015; Crosson et al., 2020). ${ }^{3}$

One way to conceptualize our model is through the inside/outside lobbying dynamic of Wolton (np). Campaign spending influences the public as outside lobbying. Policy concessions, however, directly influence the politician's implemented policy and would thus be an example of inside lobbying.

Several papers model politicians' investment in their own campaigns using an all-pay contest framework (Meirowitz, 2008; Ashworth and Bueno de Mesquita, 2009). We depart by modeling campaign contributions from interest groups, not politicians. Morton and Myerson (2012) do allow interest groups and politicians to contribute to campaigns, although they also do not analyze the effects of differential policy concessions.

Our model differs from previous models that focus on informational campaign contributions and lobbying (Cotton, 2012; Dahm and Porteiro, 2008; Schnakenberg and Turner, 2021; Bennedsen and Feldmann, 2006). We specifically focus on policy motivated interest groups, and not information. This allows us to analyze mechanisms distinct from the informational setting.

Other papers model both contributions and policy influence, but purely within a single group framework (Cotton, 2009; Judd, 2019). Our approach allows us to compare a variety of ideological alignments that are not feasible with only one interest group. To the extent that prior work has investigated the link between campaign contributions and access, it tends to presume that contributions would buy access. In our formulation, the politician can use concessions to buy electorally valuable contributions.

This paper also contributes to the literature on interest group ideology and its policy implications. Bonica $(2013,2014)$ develop procedures to measure interest group ideology based donations to candidates, while Lorenz et al. (2020) use the MapLight position taking database to construct their own scores of interest groups ideology. McKay (2010) and Thieme (2020) show that more extreme interest groups spend more

\footnotetext{
${ }^{3}$ We focus on visible contributions. Another stream of the literature focuses on the implications for transparency of dark money and shadow lobbying. See Dimmery and Peterson (2016); Strickland (2019) for more.
} 
than moderate groups on campaigns. Hirsch et al. (2020) finds that lobbyists become more selective in which special interest groups to represent as the lobbyists become more ideologically aligned with politicians. Brunell (2005) shows that while interest groups often give to both parties, their sincere giving is only targeted to one party. Grumbach and Pierson (2019) finds that when looking at "grey money" giving, corporations are more conservative than when one only looks at fully transparent PAC contributions. In our concluding discussion, we discuss several insights of the model for empirical research in this field and, in particular, efforts to scale jointly politicians and interest groups.

The empirical literature has also been mixed with regards to the effectiveness of political spending on election outcomes. Other papers show contributions to be very effective, in particular by increasing a candidate's chances of winning the election (Bonica, 2017; Sprick Schuster, 2020). Our model rationalizes these disparate findings. We show that changes in contributions can correlate positively and negatively with chances of winning, depending on the relative ideological position of politicians and interest groups. Furthermore, we show that groups will donate even when the politician grants no concessions.

We proceed by setting up the model, interpreting its features, and deriving equilibrium spending and concessions. We then study how changes in polarization and alignment affect policy moderation, campaign spending, and winning probability. After presenting an extension, we conclude by drawing out a number of empirical implications from the predictions of the model and offering suggestions for future research.

\section{Model Set-up}

\subsection{Preliminaries}

Three groups of players interact within the model: two interest groups $(G)$, two candidates/politicians $(P)$, and a set of voters. All players $(k)$ have an ideal point on the real line, denoted $\hat{x}_{k}$. We assume that there exists a unique median when voters are ordered by their ideal points, which we refer to as $\hat{x}_{M}$, and that players play only in weakly undominated strategies.

Of the two candidates, one is left-leaning $\left(P_{L}\right)$ and one is right-leaning $\left(P_{R}\right)$. Similarly, one interest group is left-learning $\left(G_{L}\right)$ and one is right-learning $\left(G_{R}\right)$. We call a politician-group pair $P_{j}$ and $G_{i}$ "aligned" if $i=j$ and "opposed" if $i \neq j$. We suppose $\hat{x}_{P_{L}}, \hat{x}_{G_{L}} \in\left[-\frac{3}{2},-\frac{1}{2}\right]$, and $\hat{x}_{P_{R}}, \hat{x}_{G_{R}} \in\left[\frac{1}{2}, \frac{3}{2}\right]$. We do not impose any ordering on the ideal points of aligned politician-group pairs, as the role of relative ideology among aligned pairs is a feature of interest in the analysis below. We do require that the players on the same side are closer to each other than to any of the opposing side players. The restriction that same-side players lie in a unit-length interval is a sufficient that each interest group is closer to its aligned politician than the 
opposed politician while still letting us analyze all possible ideal point orderings.

Policy is ultimately a convex combination of the winning politician's ideal point and the aligned interest group's ideal point. The final policy, denoted $\tilde{x}_{j}, j \in\{L, R\}$, is given by

$$
\tilde{x}_{j}=a_{j} \hat{x}_{G_{L}}+\left(1-a_{j}\right) \hat{x}_{P_{L}}
$$

where $a_{j}$ represents the influence an interest group has over policy should its aligned politician win.

The model has three stages: a "concessions" stage, a campaign stage, and an election stage. In the concessions stage, the candidates simultaneously decide how much policy authority (conceived of as influence over policymaking and discussed further below) to grant their aligned interest group $a_{j} \in[0,1], j \in\{L, R\}$. We assume these commitments are credible and observed by all players. Hence, the location of the final policy should candidate $P_{j}$ win is understood by the voter to be $\tilde{x}_{j}$, as specified in (1).

In the campaign stage, the interest groups simultaneously make spending decisions. We define $s_{j} \geq$ $0, j \in\{L, R\}$ as the contribution of interest group $j$ to its aligned politician, which ultimately translates to candidate valence. We discuss the assumption that interest groups contribute only to aligned politicians below.

In the election stage, all voters select one of the two candidates. The candidate that wins the majority of the votes subsequently takes office and enacts the final policy as given by (1). Given the assumption that players adopt weakly undominated strategies, the unidimensional policy space, and the additive nature of valence in the utility functions specified below, we may restrict attention to the median voter's decision.

Before the election, the candidates and interest groups are uncertain as to the location of the median ideal point, which is revealed after the concessions and contribution stages. When they take their actions, the candidates and interest groups know only each other's ideal points and past actions, and that $\hat{x}_{M} \sim$ $U\left(-\frac{1}{2}, \frac{1}{2}\right)$. This information structure is common knowledge.

All players' incur quadratic loss in the distance of final policies from the players' ideal points. In addition to policy, voters also value politician valence. The median voter's utility if politician $j$ wins the election with valence $v_{j}$ and implements $\tilde{x}_{j}$ is given by

$$
U_{M}\left(\tilde{x}_{j}, v_{j} ; \hat{x}_{M}\right)=-\left(\tilde{x}_{j}-\hat{x}_{M}\right)^{2}+v_{j}
$$

The cost of producing valence $v_{j}$ is $s_{j}=v_{j}^{2} / 2$. All things equal, interest groups prefer to spend less. Interest group $i$ 's utility from producing valence $v_{i}$ and candidate $j$ (where $j$ may or may not equal $i$ ) 
implementing $\tilde{x}_{j}$ is given by

$$
\begin{aligned}
U_{G_{i}}\left(\tilde{x}_{j}, v_{i} ; \hat{x}_{G_{i}}\right) & =-\left(\tilde{x}_{j}-\hat{x}_{G_{i}}\right)^{2}-s_{i}\left(v_{i}\right) \\
& =-\left(\tilde{x}_{j}-\hat{x}_{G_{i}}\right)^{2}-v_{i}^{2} / 2 .
\end{aligned}
$$

Politicians care only about policy. While valence investments by the aligned interest group affect a politician's probability of winning, it has direct benefit for the politician. The utility function representing politician $j$ 's preferences from candidate $i$ (which, again, may or may not be equal to $j$ ) winning and implementing final policy $\tilde{x}_{i}$ is given by

$$
U_{P_{j}}\left(\tilde{x}_{i} ; \hat{x}_{P_{j}}\right)=-\left(\tilde{x}_{i}-\hat{x}_{P_{j}}\right)^{2}
$$

\subsection{Reframing}

Before proceeding to interpret and analyze the model, we propose an alternative yet equivalent set of parameters to describe the exogenous features of the model. Specifically, we recast the collection of ideal points in terms of fundamental descriptors of ideological (dis)agreement between pairs of actors in the model. Reframing the model as such offers a clearer and more enlightening approach to conducting the equilibrium and comparative statics analyses than is achieved by considering changes in individual ideal points, especially when it comes to generating empirically relevant predictions.

We employ the following definitions:

$$
\begin{aligned}
\pi & =\hat{x}_{P_{R}}-\hat{x}_{P_{L}} \\
\mu & =\left(\hat{x}_{P_{R}}+\hat{x}_{P_{L}}\right) / 2 \\
\epsilon_{L} & =\hat{x}_{P_{L}}-\hat{x}_{G_{L}} \\
\epsilon_{R} & =\hat{x}_{G_{R}}-\hat{x}_{P_{R}}
\end{aligned}
$$

Political polarization, or the ideological distance between the candidates/politicians, is captured by $\pi$. The partisan leaning of the electorate may be gleaned from $\mu$, which is negative if the expected ideal point of the median voter is closer to the right-leaning candidate's ideal point than it is to the left-leaning candidate's ideal point (and vice-versa for positive $\mu$ ). The extremism of an interest group relative to its aligned politician extremism is given by $\epsilon_{L}$ for the left-leaning actors and $\epsilon_{R}$ for the right-leaning actors. A positive $\epsilon_{L}$ (resp., $\left.\epsilon_{R}\right)$ implies the left interest group is more extreme than the left politician, i.e., $\hat{x}_{G_{L}}<\hat{x}_{P_{L}}$ (resp., $\hat{x}_{G_{R}}>\hat{x}_{P_{R}}$ ). 
A negative value of $\epsilon_{L}$ or $\epsilon_{R}$ implies the interest group is more moderate than its aligned politician. A value of zero implies perfect interest group-politician alignment, and misalignment of an interest group-politician pair increases in the absolute value of $\epsilon_{L}$ or $\epsilon_{R}$.

These quantities constitute a more substantive description of the political landscape than do the individual ideal points. Furthermore, they correspond directly to empirical quantities of interest. As will become apparent below, this recharacterization also greatly simplifies the analysis. Note also that all of the ideal points may be recovered from the alternative parameters, so no generality or richness has been lost via the reparameterization.

As the remainder of the paper makes use of the parameters above, it is worth making explicit the translation of the restrictions on ideal points. Because the ideal points of politicians and interest groups were assumed to lie on either side of the interval [-1/2,1/2], it follows that $\pi+\epsilon_{L}+\epsilon_{R}=\hat{x}_{G_{R}}-\hat{x}_{G_{L}} \geq 1$. The partisan leaning of the electorate also sets a somewhat stronger lower bound on polarization, specifically, $\pi \geq 1+2|\mu|$. Finally, in this formulation, final policies are:

$$
\begin{aligned}
& \tilde{x}_{L}=\hat{x}_{P_{L}}-a_{L} \epsilon_{L} \\
& \tilde{x}_{R}=\hat{x}_{P_{R}}+a_{R} \epsilon_{R} .
\end{aligned}
$$

Figures 4 and 5 show examples of various polarization and alignment possibilities.

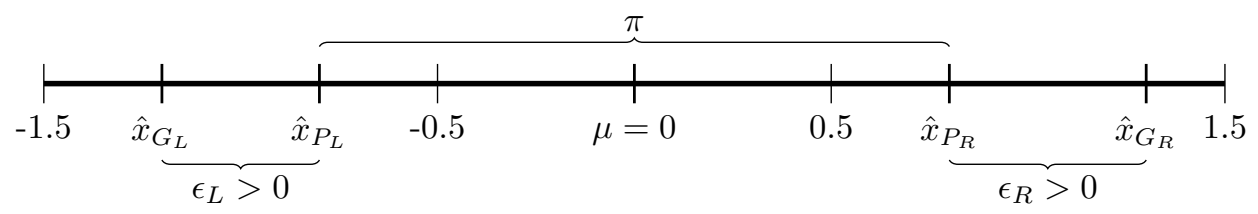

Figure 4: Interest groups relatively more extreme than their aligned politicians

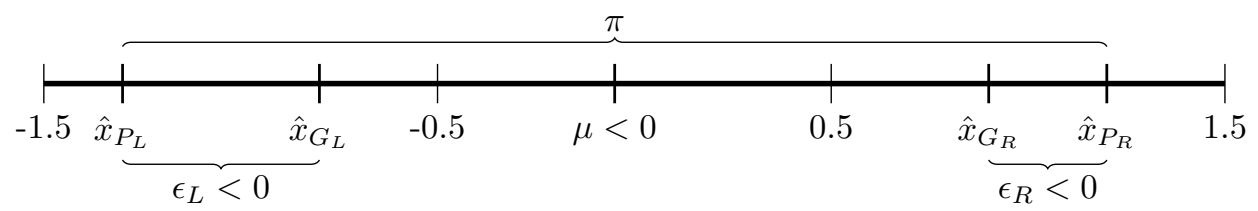

Figure 5: Interest groups relatively more moderate than their aligned politicians

As noted above, the parameters $\epsilon_{L}$ and $\epsilon_{R}$ capture how extreme the interest groups are relative to their aligned politicians. For $\epsilon_{L}<0$ or $\epsilon_{R}<0$, we say the interest group is relatively less extreme or relatively more moderate than its aligned politician. Bearing in mind that the extremism measured by $\epsilon_{L}$ and $\epsilon_{R}$ is relative to the extremism of an aligned politician, we often employ the shorthand of referring to increases 
in $\epsilon_{L}$ and $\epsilon_{R}$ as interest groups becoming relatively more extreme, as greater group relative extremism, or even simply as greater extremism. It is worth emphasizing that, holding groups' relative extremism fixed, increases in polarization necessitate movement in (at least one of) the interest group ideal points. Finally, we say a group and its aligned politician are more aligned as $\epsilon_{L}$ or $\epsilon_{R}$ approach zero from above or below.

\subsection{Interpretation of the Model's Features}

Before we proceed to the analysis, we comment on our modeling assumptions and interpretation of the game. First, we assume that the politician receives no extra rents from being elected to office, but we explain below why all of the results would hold in their current form in the presence of office motivation. We also assume that the absolute values of interest group and politician ideal points lie within $\left[\frac{1}{2}, \frac{3}{2}\right]$, such that same-side groups and candidates are always closer ideologically to one another than to a group or candidate on the other side of the median. This also ensures that both politicians have a positive probability of winning the election, and thus that there is interesting strategic behavior to analyze.

An underlying assumption is that the politician is able to commit to providing an interest group a level of concessions, even once the election has completed. We view this assumption as a simplification capturing a long-term relationship within a one-period model. Groll and Ellis (2017) provide one model where concessions driven by access are sustained in a long-term setting.

We assume that the voter observes the concessions and understands the effect on final policies. One way to think about this assumption is that it is similar to a model where candidates make binding platform commitments but promise concessions before the platform announcement. The voter does not have to actually observe of the concessions stage for the same effects to occur.

More substantively, we assume that politicians only give concessions to aligned interest groups, the main implication of which is that interest groups only contribute to their aligned candidates. This matches empirical work that finds groups give more to aligned candidates, especially in close races (Barber et al., 2017). We also focus on the subclass of interest groups that engage in both lobbying and campaign contributions. This is a highly relevant group; even though they comprise a minority of all interest groups, they spend a majority of special interest money (Lake, 2015; Crosson et al., 2020). ${ }^{4}$

Campaign spending in the model improves the electoral chances of a candidate. This may be through direct contributions, advertising, or other investments. ${ }^{5}$ The model captures this by supposing interest group expenditures before the election increase a candidate's valence. The group dislikes spending money as the opportunity cost of money spent during the campaign stage includes all manner of other activities in which

\footnotetext{
${ }^{4}$ We abstract away from lobbyists targeting specific legislators other than the aligned candidate. See de Figueiredo and Cameron (2019); You (2020) for more on targeting.

${ }^{5}$ For example, Miller (2019) shows that outside groups are more likely to produce negative attack ads.
} 
interest groups engage, such as conducting membership drives, increasing salaries, or even additional fund raising. While campaign spending translates into a valence attribute, we do not want to over interpret the how much utility the voter receives from campaign spending. Instead, we view spending and valence as technologies that improve the probability of winning the election. The campaign stage draws upon the uncertain median voter technology first used in Calvert (1985) and extended in Groseclose (2001), amongst others.

Our modeling of policy concessions captures a reduced-form approach to interest group influence on final policy. Specifically, we suppose a final policy will be a convex combination of the interest group's ideal point and the politician's ideal point, where the weight placed on the interest group's ideal point increases in the concessions it receives from the politician. This account is consistent with a legislative subsidy in the sense of Hall and Deardorff (2006) or informational lobbying in the sense of Bennedsen and Feldmann (2006). Interest groups can influence final policy with bill-writing, advice, expertise, and other non-monetary support (Kroeger, 2020). Through such efforts, lobbyists and politicians move policy closer to their own ideal points. Further, this approach is compatible with the equilibria of micro-founded models of lobbying such as Duggan and Gao (2019) and Grossman and Helpman (2001).

The reparamterization of the model in terms of $\pi, \mu, \epsilon_{L}$, and $\epsilon_{R}$ serves not only to streamline the analysis and exposition but also to emphasize the most empirically relevant relationships of the model. When scholars employ measures of ideology in empirical work, the interest is generally in ordinal properties across actors or for a given actor over time. (On which side of the median does a legislator lie? Is a legislator growing more extreme over time?) Indeed, empirical work largely seeks to purge from its analyses any variation that is an artifact of scaling procedures. The reparameterization focuses our analysis on exactly the variation an empiricist would seek to isolate: changes in the distance between politicians' ideological stances, changes in the relative ideological positions of groups and aligned politicians, and changes in the partisan leaning of a constituency.

Our framework allows a unified discussion of groups' relative extremism $\left(\epsilon_{L}, \epsilon_{R}\right)$ and alignment $\left(-\left|\epsilon_{L}\right|,-\left|\epsilon_{R}\right|\right)$ with same-side politicians. Further, we are able to disentangle the role of interest group extremism and interest group positioning relative to nearby politicians by "controlling for" levels of polarization. For example, both a group and its aligned politician could be very extreme in an absolute sense, but if they are perfectly aligned and $\epsilon_{L}=0$, then the left group is neither extreme nor moderate relative to its aligned politician. 


\section{Equilibrium Analysis}

Because the median voter's ideal point is revealed before the voting stage, the appropriate solution concept is Subgame Perfect Equilibrium (hereafter just "equilibrium"). Therefore, we proceed backwards through the stages. The electoral stage provides the probability that a given candidate wins given the strategies adopted by candidates and interest groups.

\subsection{Analysis of the Electoral Stage}

The voter votes for $L$ if:

$$
\begin{aligned}
-\left(\tilde{x}_{L}-\hat{x}_{M}\right)^{2}+v_{L} & >-\left(\tilde{x}_{R}-\hat{x}_{M}\right)^{2}+v_{R} \\
\frac{\tilde{x}_{R}+\tilde{x}_{L}}{2}+\frac{v_{L}-v_{R}}{2\left(\tilde{x}_{R}-\tilde{x}_{L}\right)} & >\hat{x}_{M} .
\end{aligned}
$$

Employing the expressions for the final policies given in 6 and 7, the condition for the voter to strictly prefer $L$ simplifies to: ${ }^{6}$

$$
\underbrace{\mu+\frac{a_{R} \epsilon_{R}-a_{L} \epsilon_{L}}{2}}_{\text {Policy Effect }}+\underbrace{\frac{v_{L}-v_{R}}{2\left(\pi+a_{R} \epsilon_{R}+a_{L} \epsilon_{L}\right)}}_{\text {Spending Effect }} \geq \hat{x}_{M} .
$$

The quantity on the left-hand side of (8) is larger the more the district leans left (first term). The more extreme the right interest group is and the more policy concessions it is given - relative to the extremity of the left interest group and the policy concessions it is given - the more attractive the left candidate becomes (second term). The left-hand side expression also increases in the valence for $L$ and decreases in the valence for $R$, but this effect is muted by polarization and policy concessions to interest groups more extreme than their aligned politicians (third term). Note that the requirement of ceteris paribus in, e.g., the statement regarding the second term is that the denominator on the third term remain fixed. These comparative statics on the voter's decision problem, however, highlight the intuitive implications in terms of politically relevant quantities that the transformed parameter space yields.

Recalling $\hat{x}_{M} \sim U(-1 / 2,1 / 2)$, we may translate the voter's cutoff decision into the probabilities that

\footnotetext{
${ }^{6}$ Note that $\frac{\tilde{x}_{R}+\tilde{x}_{L}}{2}=\hat{x}_{P_{R}}+a_{R} \epsilon_{R}+\hat{x}_{P_{L}}-a_{L} \epsilon_{L}=\mu+\frac{a_{R} \epsilon_{R}-a_{L} \epsilon_{L}}{2}$ and that $\tilde{x}_{R}-\tilde{x}_{L}=\hat{x}_{P_{R}}+a_{R} \epsilon_{R}-\left(\hat{x}_{P_{L}}-a_{L} \epsilon_{L}\right)=$ $\pi+a_{R} \epsilon_{R}+a_{L} \epsilon_{L}$
} 
each candidate wins the election:

$$
\begin{aligned}
& \operatorname{Pr}\left(P_{L} \text { wins }\right)=\mu+\frac{a_{R} \epsilon_{R}-a_{L} \epsilon_{L}}{2}+\frac{v_{L}-v_{R}}{2\left(\pi+a_{R} \epsilon_{R}+a_{L} \epsilon_{L}\right)}+\frac{1}{2} \\
& \operatorname{Pr}\left(P_{R} \text { wins }\right)=\frac{1}{2}-\mu+\frac{a_{L} \epsilon_{L}-a_{R} \epsilon_{R}}{2}+\frac{v_{R}-v_{L}}{2\left(\pi+a_{R} \epsilon_{R}+a_{L} \epsilon_{L}\right)} .
\end{aligned}
$$

As the interest groups increase campaign spending, they improve the electoral prospects of their preferred candidate. Of course, this all else equal statement may not hold in equilibrium. We turn to equilibrium spending next.

\subsection{Analysis of optimal campaign spending}

The derivation of each interest group's optimal campaign contribution is straightforward. For simplicity, and continuity with the voter's and the candidates' decision problems, we proceed in terms of the valence procured for a group's aligned candidate. We may then back out the equilibrium campaign spending. The expected utility for the left group is given by: ${ }^{7}$

$$
\begin{aligned}
\mathbb{E} U_{G_{L}}\left(v_{L}\right)= & -\operatorname{Pr}\left(P_{L} \text { wins } \mid v_{L}\right)\left(\tilde{x}_{L}-\hat{x}_{G_{L}}\right)^{2}-\operatorname{Pr}\left(P_{R} \text { wins } \mid v_{L}\right)\left(\tilde{x}_{R}-\hat{x}_{G_{L}}\right)^{2}-\frac{v_{L}^{2}}{2} \\
= & -\left(1+2 \mu+a_{R} \epsilon_{R}-a_{L} \epsilon_{L}+\frac{v_{L}-v_{R}}{\pi+a_{R} \epsilon_{R}+a_{L} \epsilon_{L}}\right) \frac{\left(\left(1-a_{L}\right) \epsilon_{L}\right)^{2}}{2} \\
& -\left(1-2 \mu+a_{L} \epsilon_{L}-a_{R} \epsilon_{R}+\frac{v_{R}-v_{L}}{\pi+a_{R} \epsilon_{R}+a_{L} \epsilon_{L}}\right) \frac{\left(\pi+a_{R} \epsilon_{R}+\epsilon_{L}\right)^{2}}{2}-\frac{v_{L}^{2}}{2} .
\end{aligned}
$$

The expected utility for the right group is given by: ${ }^{8}$

$$
\begin{aligned}
\mathbb{E} U_{G_{R}}\left(v_{R}\right)= & -\operatorname{Pr}\left(P_{R} \text { wins } \mid v_{R}\right)\left(\tilde{x}_{R}-\hat{x}_{G_{R}}\right)^{2}-\operatorname{Pr}\left(P_{L} \text { wins } \mid v_{R}\right)\left(\tilde{x}_{L}-\hat{x}_{G_{R}}\right)^{2}-\frac{v_{R}^{2}}{2} \\
= & -\left(1-2 \mu+a_{L} \epsilon_{L}-a_{R} \epsilon_{R}+\frac{v_{R}-v_{L}}{\pi+a_{R} \epsilon_{R}+a_{L} \epsilon_{L}}\right) \frac{\left(\left(1-a_{R}\right) \epsilon_{R}\right)^{2}}{2} \\
& -\left(1+2 \mu+a_{R} \epsilon_{R}-a_{L} \epsilon_{L}+\frac{v_{L}-v_{R}}{\pi+a_{R} \epsilon_{R}+a_{L} \epsilon_{L}}\right) \frac{\left(\pi+a_{L} \epsilon_{L}+\epsilon_{R}\right)^{2}}{2}-\frac{v_{R}^{2}}{2} .
\end{aligned}
$$

Maximizing each group's expected utility with respect to the valence it produces yields the equilibrium levels:

$$
\begin{aligned}
& v_{L}^{*}=\frac{\pi+\left(2-a_{L}\right) \epsilon_{L}+a_{R} \epsilon_{R}}{2} \\
& v_{R}^{*}=\frac{\pi+\left(2-a_{R}\right) \epsilon_{R}+a_{L} \epsilon_{L}}{2} .
\end{aligned}
$$

\footnotetext{
${ }^{7}$ Note that $-\left(\tilde{x}_{L}-\hat{x}_{G_{L}}\right)^{2}=-\left(\left(1-a_{L}\right) \epsilon_{L}\right)^{2}$ and that $-\left(\tilde{x}_{R}-\hat{x}_{G_{L}}\right)^{2}=-\left(\hat{x}_{P_{R}}+a_{R} \epsilon_{R}-\hat{x}_{G_{L}}\right)^{2}=-\left(\pi+a_{R} \epsilon_{R}+\epsilon_{L}\right)^{2}$.

${ }^{8}$ Note that $-\left(\tilde{x}_{R}-\hat{x}_{G_{R}}\right)^{2}=-\left(\left(a_{R}-1\right) \epsilon_{R}\right)^{2}=-\left(\left(1-a_{R}\right) \epsilon_{R}\right)^{2}$ and that $-\left(\tilde{x}_{L}-\hat{x}_{G_{R}}\right)^{2}=-\left(\hat{x}_{P_{L}}-a_{L} \epsilon_{L}-\hat{x}_{G_{R}}\right)^{2}=$ $-\left(-\pi-a_{L} \epsilon_{L}-\epsilon_{R}\right)^{2}=-\left(\pi+a_{L} \epsilon_{L}+\epsilon_{R}\right)$.
} 
Equilibrium spending is then $s_{i}^{*}=\frac{\left(v_{i}^{*}\right)^{2}}{2}, i=L, R$.

Equations 11 and 12 give the equilibrium valence levels. We can then use these equations to see how the valence produced via campaign spending responds to changes in polarization and alignment.

Proposition 1. The valence a group produces for its aligned candidate is increasing in polarization, increasing in its extremism relative to its aligned politician, and weakly increasing in the opposing group's extremism relative to that group's aligned politician.

A relatively more extreme (resp., moderate) group produces less (resp., more) valence as its aligned politician promises it greater policy concessions and more (resp., less) as the opposing politician grants greater policy concessions to its aligned group if that opposing group is relatively more extreme (resp., moderate) than the opposing politician.

Holding everything else fixed, increasing polarization makes losing the election worse for the interest group as the opposing policy is now farther away, inducing the group to spend more as polarization increases. This only holds for relatively moderate groups if preferences are represented by a concave utility function. The same force holds as the interest group becomes more extreme and as the opposing interest group becomes more extreme. Proposition 1 also describes how policy concessions affect spending by both interest groups. Specifically, concessions to a more moderate interest group increases valence produced by the aligned group (winning is more desirable) and decreases valence produced by the opposing group (losing is less undesirable). Conversely, granting concessions to a more extreme interest group decreases valence produced by the aligned group (concessions in this case produces a more divergent final policy that is less likely to win) and increases valence produced by the opposing group (losing is more undesirable). The downstream effects on spending are central to the discussion of concessions below.

\subsection{Analysis of the concessions decision}

With the optimal campaign expenditures in hand, we may turn to the concessions granted by candidates to interest groups. Before delving into the analysis of equilibrium concessions decisions, it is enlightening to step back from the decision around granting concessions in order to consider the candidates' concessions decisions as a form of proposing policies in a Downsian setting, albeit with some important constraints. At heart, two policy-motivated candidates engage in unidimensional policy competition with uncertainty around the ideological location of the median voter. We know from previous work that in this setting, the full convergence result from models without policy motivation and/or uncertainty is weakened somewhat but not entirely (Duggan, 2008). How do the particular features of this model interact with this baseline of some-but-not-full policy convergence? 
The restriction that final policies be a convex combination of aligned politician and group ideal points limits the extent to which - through the concessions decision - candidates can achieve convergence. When a group is more extreme than its aligned politician $\left(\epsilon_{L}, \epsilon_{R}>0\right)$, concessions represent divergence in policy. When a group is less extreme than its aligned politician $\left(\epsilon_{L}, \epsilon_{R}<0\right)$, granting concessions constitutes the candidates proposing more convergent policies than their ideal points. Setting aside this constraint on convergence, however, the more fundamental question is whether the downstream effect of concessions on campaign contributions weakens or strengthens the tendency towards convergence of policy competition.

Granting concessions has three effects from the perspective of a candidate. First, it makes policy less desirable. Instead of the winning policy at the candidate's ideal point, it will be somewhere in between the candidate's and interest group's ideal points. Second, it affects how the median voter evaluates candidates. Granting concessions to a moderate interest group will make the politician more competitive as policy is pulled towards the median voter. Granting concessions to an extreme interest group, however, will make the politician less competitive as policy is moved away from the median voter. Third, it affects the opposing group's campaign spending decision. Granting concessions to a moderate group induces the opposing group to spend less while granting concessions to an extreme group induces the opposing group the spend more.

Proposition 2. In equilibrium, when a candidate is relatively more moderate than its aligned interest group, the candidate provides no policy concessions to the aligned group (i.e., $a_{L}^{*}=0$ if $\epsilon_{L}<0, a_{R}^{*}=0$ if $\epsilon_{R}<0$ ).

When a candidate is relatively more extreme than its aligned interest group, the candidate provides full policy concessions to the aligned group (i.e., $a_{L}^{*}=1$ if $\epsilon_{L}>0, a_{R}^{*}=1$ if $\epsilon_{R}>0$ ).

Proposition 1 establishes that contributions respond to concessions in such a way as to strengthen candidates' incentives to produce - via concessions - more convergent policies. The baseline pressures from policy competition deter candidates from granting concessions to a more extreme politician as it produces a less attractive final policy for both the candidate and the median voter. Proposition 1 then tells us greater concessions to more extreme groups puts downward pressure on campaign contributions, and it draws more spending from the opposing interest group, eliminating any motivation to provide concessions to a more extreme interest group that a candidate might have had. As this suggests, politicians will never grant concessions to more extreme groups.

When aligned interest groups are more moderate than politicians, greater concessions drives up friendly campaign contributions and drives down contributions to the opposing candidate. This adds to candidates' baseline motivation to "propose" a moderate policy. Indeed, the effects on campaign contributions are so strong that candidates will grant full concessions to a more moderate interest group, achieving the most convergent policy they can. Candidates' optimal concessions decisions are thus a step function with respect 
to the relative extremism of their aligned interest group. Proposition 2 formalizes this intuition.

One might wonder if these results are due to focusing on policy motivated politicians instead of office motivated politicians. All of our results are robust to adding in office motivation. In fact, office motivation makes the desire to moderate even stronger. By focusing purely on policy motivated politicians, we have effectively stacked the deck against finding the that moderate players have full authority over policy location.

\section{Comparative Statics}

\subsection{Final policy moderation}

The implications of the proposition above for the locations of final policies is stark. The voter expects that final policies will be the ideal point of the more moderate member within each interest group-candidate pair. Specifically, where $\tilde{x}_{j}^{*}$ is the final policy $P_{j}$ will enact in equilibrium if elected, $\tilde{x}_{R}^{*}=\min \left\{\hat{x}_{G_{R}}, \hat{x}_{P_{R}}\right\}$ and $\tilde{x}_{L}^{*}=\max \left\{\hat{x}_{G_{L}}, \hat{x}_{P_{L}}\right\}$.

One measure of the effect of polarization and extremism of politicians and interest groups on the policymaking process is the distance between the policies opposing candidates would enact. If a candidate is relatively more extreme than its aligned interest group $\left(\epsilon_{L}, \epsilon_{R}<0\right)$, final policy becomes more extreme on that side of the median only if the group becomes more extreme. If a candidate is more relatively more moderate than its aligned interest group $\left(\epsilon_{L}, \epsilon_{R}>0\right)$, final policy becomes more extreme on that side if the candidate becomes more extreme. Hence, an increase in group extremism on the left or right $\left(\epsilon_{L}\right.$ or $\epsilon_{R}$ increasing) will only increase the distance between final policies if $\epsilon_{L}<0$ or $\epsilon_{R}<0$, respectively. Otherwise, there is no effect on the final policies.

A change in politician polarization $(\pi)$ holds constant group-candidate alignment $\left(\epsilon_{R}\right.$ and $\left.\epsilon_{L}\right)$ and the partisan lean $(\mu)$. Holding fixed the alignment of group-candidate pairs means any change in politician ideal

points from a change in $\pi$ has an associated change in group ideal points of equal magnitude. As such, an increase in $\pi$ not only implies that the politicians become more extreme but also that each interest group becomes more extreme, which in turn means that the distance between final policies is sure to increase in $\pi$. Corollary 1 summarizes these results.

Corollary 1. The distance between final policies strictly increases in $\pi$ and weakly increases in $\epsilon_{L}$ and $\epsilon_{R}$.

Note that from a policy perspective, voters are better off with low polarization and with moderate politicians. 
Figure 6: Relative interest group extremism and campaign spending

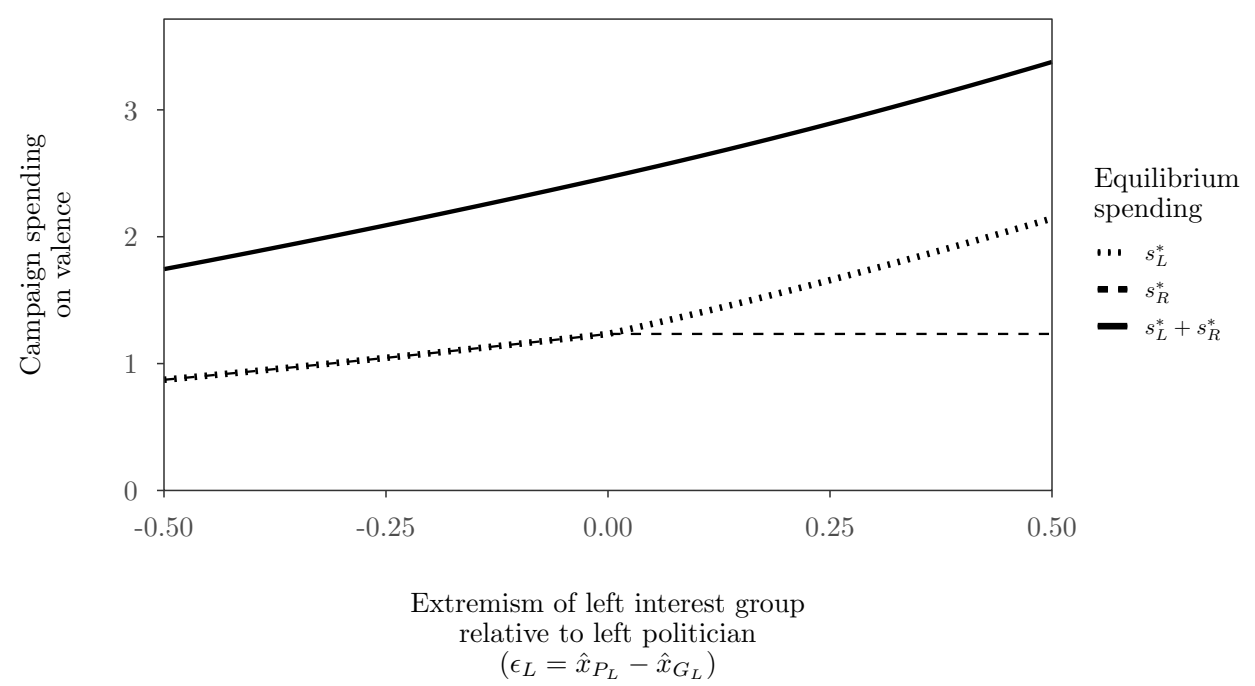

Notes: The graph depicts equilibrium spending by both interest groups, individually and together, as the left interest group grows more extreme relative to the left candidate. Polarization, the electorate's partisan lean, and the right group's relative extremism are held, respectively, at $\pi=2, \mu=0, \epsilon_{R}=0$, while the left group's extremism $\left(\epsilon_{L}\right)$ varies from -0.5 to 0.5 . Equivalently, $\hat{x}_{P_{R}}=\hat{x}_{G_{R}}=1, \hat{x}_{P_{L}}=-1$, and $\hat{x}_{G_{L}}$ varies from -0.5 to -1.5 .

\subsection{Spending and valence}

We begin to analyze interest group spending by asking how a group's spending responds to changes in the opposing group's extremism. Consider interest group $G_{i}$ and the opposing interest group $G_{j}, j \neq i$. When $G_{j}$ is less extreme than its aligned politician, $P_{j}$, then as $G_{j}$ grows more extreme (more aligned with $P_{j}$ ), the anticipated policy on that side of the median grows more extreme. This makes losing worse for $G_{i}$ and thus induces $G_{i}$ to spend more on the valence of its aligned candidate, $P_{i}$. Once $G_{j}$ is more extreme than $P_{j}$, however, additional extremism has no effect on policy due to $G_{j}$ no longer receiving any concessions. Further, because interest group spending does not directly depend on opposing group spending in this model, ${ }^{9} G_{i}$ does not spend more as $G_{j}$ grows even more extreme relative to $P_{j}$.

This pattern of spending may be observed in Figure 6 (setting $j=L$ and $i=R$ ). Equilibrium spending by $G_{R}, s_{R}^{*}$, increases in $\epsilon_{L}$ for $\epsilon_{L}<0$, i.e., when $G_{L}$ is relatively less extreme than $P_{L}$. When $G_{L}$ is relatively more extreme than $P_{L}$, i.e., $\epsilon_{L}>0$, further increases in extremism have no additional effect on $s_{R}^{*}$.

The effect of changes in an interest group's own extremism on its spending is similarly the sum of several effects. For a group that is less extreme than its aligned candidate, its aligned candidate's final policy is simply the group's ideal point. However, as the group becomes more extreme, the final policy if it loses

\footnotetext{
${ }^{9}$ This stems from our use of a uniform distribution for the median voter and our assumption of an additive effect of valence.
} 
becomes less and less attractive, leading the group to spend more on its candidate's valence.

For a group that is more extreme than its aligned candidate, an increase in its extremism has no effect on its aligned candidate's final policy. The difference in utility from that final policy and the opposing side's final policy increases as the group grows more extreme, ${ }^{10}$ leading the group to spend more on the campaign. In sum, increases in a group's own extremism lead it to spend more, regardless of whether it is relatively less or more extreme than its aligned candidate, depicted in Figure 6 by $s_{L}^{*}$ strictly increasing in $\epsilon_{L}$.

Before turning to the effect of increases in candidate polarization, it is worthwhile to summarize the insights about spending thus far. An interest group's spending strictly increases in its own extremism. An interest group's spending weakly increases in opposing group extremism, strictly increasing when the opposing group is relatively less extreme than that group's aligned candidate and constant for changes in the extremism of an opposing group that is relatively more extreme than its aligned candidate.

An increase in a candidate's extremism, or even polarization between candidates, would usually not necessarily imply that the final policies change location - it could be that both groups are more moderate than their aligned candidates. In our setting, however, changes in polarization hold all else equal, where all else equal entails the alignment between each politician and its aligned group. In our setup, then, an increase in polarization does necessarily imply that distance between final policies also increases. Greater polarization in anticipated final policies increases the benefit to a group of having its aligned candidate win rather than the opposing candidate win. Hence, as Figure 7 depicts, spending by both groups increases as polarization increases, regardless of whether they are relatively more or less extreme than their aligned politician.

Corollary 2 below distills the topline comparative statics of the model with respect to spending. It omits some of the nuance above, but highlights the results that we make most use of below. Specifically, spending monotonically increases as groups grow more extreme and politicians more polarized. ${ }^{11}$

Corollary 2. Equilibrium campaign spending - both total and by each interest group - increases in $\epsilon_{L}, \epsilon_{R}$, and $\pi$.

Recalling Corollary 1, spending and the distance between final policies respond similarly to changes in polarization and relative interest group extremism. More specifically, spending outcomes and the distance between final policies are both strictly increasing in $\pi$ and weakly increasing in groups' relative extremism, $\epsilon_{L}$ and $\epsilon_{R}$.

\footnotetext{
${ }^{10}$ This stems from the concavity of the quadratic loss function used for the policy component of groups' utilities.

${ }^{11}$ In an auction setting, Montagnes and Van Weelden (2019) also show how polarization can increase spending.
} 
Figure 7: Politician polarization and campaign spending

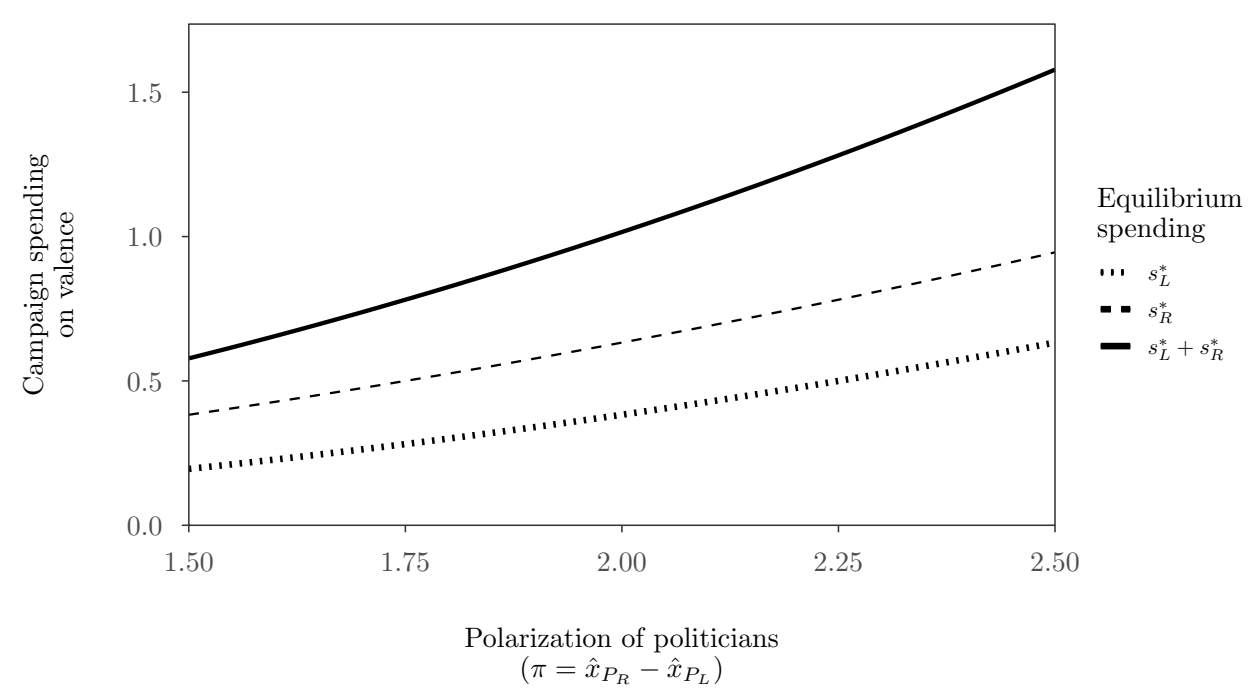

Notes: The graph depicts equilibrium spending by both interest groups, individually and together, as the politicians grow more polarized. Polarization varies from 1.5 to 2.5 , while the electorate's partisan lean, the right group's relative extremism, and the left group's relative extremism are held, respectively, at $\mu=0, \epsilon_{R}=0.25$, and $\epsilon_{L}=-0.25$. Equivalently, $\hat{x}_{G_{R}}=0.25+\hat{x}_{P_{R}}, \hat{x}_{G_{L}}=\hat{x}_{P_{L}}+0.25$, and $\hat{x}_{P_{R}}=-\hat{x}_{P_{L}}$ varies from 0.75 to 1.25. We observe both interest groups' spending increasing in polarization, and the group more extreme relative to its aligned politician spends more.

\subsection{Probability of winning}

We next ask how the probability that a given politician wins responds to changes in the alignment of the various players. The first result is striking in and of itself, and even more so when juxtaposed with other comparative statics. The more aligned an interest group-candidate pair, the less likely it is to win. This holds fixed the polarization parameter and the opposing side's group-candidate alignment. Conversely, the less aligned a group is with a candidate - in either direction - the more likely its candidate is to win in equilibrium.

Given that one might plausibly think that alignment connotes solidarity and electoral strength, the result above merits some unpacking. A group that is relatively more moderate than its aligned candidate will be given full concessions (Proposition 2). As such a group becomes even more moderate (one form of being less aligned with its candidate), the final policy grows more moderate. Even though the group spends less on the campaign (Corollary 2), the moderation of final policy still increases its aligned candidate's chance of winning.

A group that is more extreme than its aligned candidate will not be given any concessions (Proposition 2). Policy will not change if such a group becomes more extreme (the other form of being less aligned). The 
group will spend more, however, and its opposing interest group's spending will remain constant (Corollary 2). With no change to a candidate's final policy, more campaign spending by the candidate's aligned group, and no change in campaign spending by the opposing side, the candidate's chances of winning increase as an already more extreme group grows more extreme.

While a group's equilibrium spending increases monotonically in its extremism, its aligned candidate's equilibrium probability of winning responds non-monotonically to increases in the group's extremism (i.e., increases in $\epsilon_{L}$ or $\epsilon_{R}$ ). The equilibrium probability of a group's aligned candidate winning falls as a relatively more moderate group becomes more aligned with its candidate (e.g., $\epsilon_{L} \uparrow 0$ or $\epsilon_{R} \uparrow 0$ ) but rises as a relatively more extreme group $\left(\epsilon_{L}>0\right.$ or $\left.\epsilon_{R}>0\right)$ grows more extreme.

Corollary 3. The equilibrium probability a politician wins is decreasing as alignment with their same-side interest group increases $\left(\epsilon_{L} \uparrow 0\right.$ or $\epsilon_{L} \downarrow 0$ for $\operatorname{Pr}\left(P_{L}\right.$ wins $), \epsilon_{R} \uparrow 0$ or $\epsilon_{R} \downarrow 0$ for $\operatorname{Pr}\left(P_{R}\right.$ wins $\left.)\right)$.

As an interest group that is relatively more moderate than its aligned politician grows more aligned (e.g., $\epsilon_{L}$ increasing for $\epsilon_{L}<0$ ), equilibrium spending increases but the equilibrium probability the aligned politician wins falls.

Figure 8 illustrates the comparison in Corollary 3. As the left-side interest group becomes more extreme - and the alignment of the left interest group-politician pair increases and then decreases - the probability that $P_{L}$ wins falls and then rises. Over this same range of $\epsilon_{L}$, however, spending increases monotonically. For $\epsilon_{L}>0$, increases in $\epsilon_{L}$ lead $G_{L}$ to spend more and $P_{L}$ to win with higher probability. For $\epsilon_{L}<0$, increases in $\epsilon_{L}$ lead $G_{L}$ to spend more but $P_{L}$ to win with lower probability.

Because winning is zero-sum, Corollary 3 encodes the comparative statics for the way in which a given side's probability of winning responds to changes in the opposing side's alignment. A given side becomes more likely to win as the opposing interest group-politician pair grows more aligned, as win probability is decreasing in own-alignment. Changes in a side's spending and win probability will be weakly correlated in relation to opposing-side alignment. For example, if $\epsilon_{R}<0$, increases in $\epsilon_{R}$ lead to greater spending by $G_{L}$ and a higher win probability for $P_{L}$. When $\epsilon_{R}>0$, further increases in $\epsilon_{R}$ lead to a lower win probability for $P_{L}$. However, increases in $\epsilon_{R}>0$ have no effect on $G_{L}$ 's equilibrium spending, $s_{L}^{*}$.

Finally, rising polarization increases the win probability of the politician with the less relatively-extreme aligned interest group. That is, if $\epsilon_{L}<\epsilon_{R}$ (resp., >), an increase in $\pi$ will weakly increase (resp., decrease) the equilibrium probability that $P_{L}$ wins the election, and vice versa. Since spending by both groups is increasing in the polarization of the politicians, a group's spending and the probability its aligned candidate wins may move in the same direction or opposite directions as polarization changes. 
Figure 8: Comparison of the probability that $P_{L}$ wins and $G_{L}$ 's campaign spending
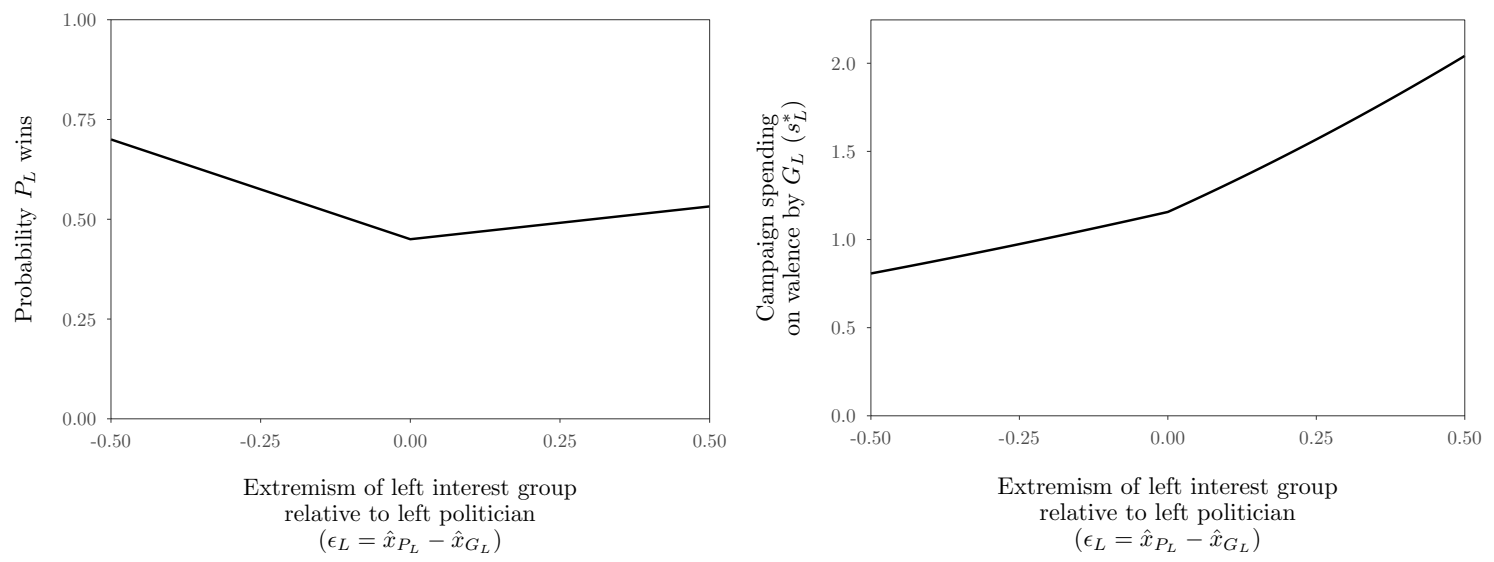

Notes: The graph on the left depicts the equilibrium probability that $P_{L}$ wins, and the graph on the right depicts equilibrium spending by $G_{L}$. The graphs plot these outcomes as a functions of the left group's relative extremism, $\epsilon_{L} \in[-0.5,0.5]$. Polarization, the electorate's partisan lean, and the right group's relative extremism are held, respectively, at $\pi=2, \mu=0$, and $\epsilon_{R}=-0.1$. Equivalently, $\hat{x}_{P_{R}}=\hat{x}_{G_{R}}=1, \hat{x}_{P_{L}}=-1$, and $\hat{x}_{G_{L}}$ varies from -0.5 to -1.5 .

\section{Extension: Candidate Selection}

In this extension, we let parties choose a candidate before the policy concessions stage. Denote the parties as $O_{R}$ and $O_{L}$ with corresponding ideal points $\hat{x}_{O_{R}}$ and $\hat{x}_{O_{L}}$. As in the baseline model, we make no restrictions as to the ordering of same-side players except that they all lie within the interval $\left[\frac{1}{2}, \frac{3}{2}\right]$ for the right side and the interval $\left[-\frac{3}{2},-\frac{1}{2}\right]$ for the left side.

The game proceeds as before except that before politicians choose the level of policy concessions to give to the interest group, the parties choose the aligned politician's ideal point from the feasible interval.

Proposition 3. Let parties choose the ideal point of the politician before the policy concession stage. Then both parties will choose the maximally moderate politician possible with $\hat{x}_{P_{R}}^{*}=\frac{1}{2}$ and $\hat{x}_{P_{L}}^{*}=-\frac{1}{2}$.

The intuition is similar as to why the politician offers policy concessions to moderate interest groups but not extreme interest groups. Moderate politicians offer the greatest probability of winning the election, and this probability increases as they become more moderate. A moderate politician will still receive campaign contributions and will also have the best chance of winning the election. While there is some policy loss to the party by nominating a candidate not located at the party's ideal point, this is outweighed by a higher probability of avoiding the other side's winning policy.

We can then characterize the optimal special interest group environment for a party given their nominating strategy: 
Corollary 4. Parties' utilities are maximized with maximally moderate politicians and maximally extreme interest groups.

Extreme interest groups are still willing to spend on campaigns even without policy concessions, and they spend more as they become more extreme. ${ }^{12}$ Therefore, the best interest group from the party's perspective is one as extreme as possible. The combination of moderate policy and extreme interest groups produces the greatest probability of winning the election and therefore the greatest probability of avoiding the other side's policy. This suggests that parties may try to form diverse coalitions over coalitions of closely ideologically aligned actors.

\section{Discussion and Conclusion}

Fundamentally, levels of concessions and campaign spending are jointly-determined equilibrium phenomena. The expectation of concessions is set in advance of any campaign spending in the model, so any campaign spending only serves to help a group's aligned candidate win the election. Despite this imposed order of play, groups will still spend on campaigns. Indeed, groups more extreme than their aligned candidate will spend on the candidate's campaign without any expectation of concessions should that candidate win the election. Our model emphasizes the role of concessions in "buying" contributions. The ex ante commitment to a level of concessions capturing the notion that concessions buys contributions as much as contributions might buy concessions.

Specifically, interest group campaign contributions increase as the benefit of having an ideologically aligned politician in office increases. Indeed, polarization between candidates is not enough to explain campaign spending or equilibrium policy location. The alignment of interest groups and candidates is a quantity as important to understanding campaign spending and policy location as the absolute polarization of politicians or groups. A central theme running throughout many of the model's results is the importance of considering an interest group's alignment with (i.e., the absolute distance from) a nearby politician as well as whether they are relatively more moderate or extreme.

The comparative statics facilitate the formation of empirical hypotheses from our model for the three outcomes discussed above. The explanatory variables would be constructed as differences in estimated ideological position between various pairs of actors. More fundamentally, the model makes clear the importance of accounting for the entire ecosystem of relevant ideological positions - not just politician or even interest group polarization but also alignment of groups and politicians. Including variables for all of the four parameters $\left(\pi, \mu, \epsilon_{L}, \epsilon_{R}\right)$ would support the all-else-equal construction of any given difference in ideal

\footnotetext{
${ }^{12}$ As above, the increase in spending is a result of assuming concave utility functions.
} 
points. Depending on the specification, first differences of estimated ideal points or the use of actor-level and period fixed effects may achieve a similar result. In so doing, and as was possible in the theoretical exposition above, it would be possible to disentangle the effects of overall political polarization and the alignment/relative positioning of groups and politicians.

The model also suggests, however, that commonly used measures of interest group ideology may not appropriately operationalize these parameters. The Bonica (2013) DIME scores are based on campaign contributions. Our model illustrates that contributions are plausibly endogenous, determined as a function of ideological distance between recipient and donor. More specifically, campaign contributions rise not in alignment but in relative extremism. In the DIME scores, estimated ideological proximity rises in contributions. As such, our model offers a prediction for the bias of such scores: the alignment of a group and a politician to whom it contributed would be overestimated (resp., underestimated) when the group is relatively more extreme (resp., moderate) than the politician.

Similarly, studies analyzing the effect of campaign spending on the probability of politicians winning the elections have often used candidate ideology as an independent variable (Gerber (1998) is one prominent example). Our model here suggests that focusing solely on individual ideology can lead researchers astray. The relative extremity of the candidates matters as much if not more than the specific extremity of a single candidate.

There are a number of potentially fruitful avenues for related work. Future work may wish to extend this rather tractable model to speak to different limitations on spending. For example, how would a limit on campaign contributions affect spending and each side's likelihood of winning when concessions determine the level of contributions?

We did not allow politicians to make campaign investments in their own campaigns, focusing only on interest group spending. How might interest group spending change when politicians may decide simply to fund their own campaigns instead of trading concessions for contributions? On this note, we also do not explicitly model the way in which campaign spending may purchase concessions, instead asking how concessions might "buy" contributions. How might spending and the number of active lobbyists in the second stage change if contributions purchased a degree of policy concessions in addition to improving electoral chances of the receiving politician? While extant work models these relationships, moving towards models with both group and candidate campaign spending, or in which concessions and spending decisions are jointly determined emerges as a likely profitable next step for this literature.

A final suggestion is to consider interest group decision making across multiple political offices. Large interest groups give to multiple races at once. How does the potential for future coalitions of politicians affect the distribution of spending across many simultaneous campaigns? The strategic interrelation of campaigns 
and lobbying points towards many avenues of interesting and worthwhile future research.

\section{References}

Ansolabehere, S., J. M. De Figueiredo, and J. M. Snyder Jr (2003). Why is there so little money in us politics? Journal of Economic perspectives 17(1), 105-130.

Ashworth, S. and E. Bueno de Mesquita (2009). Elections with platform and valence competition. Games and Economic Behavior 67(1), 191-216.

Barber, M. J., B. Canes-Wrone, and S. Thrower (2017). Ideologically sophisticated donors: Which candidates do individual contributors finance? American Journal of Political Science 61(2), 271-288.

Bennedsen, M. and S. E. Feldmann (2006). Informational lobbying and political contributions. Journal of Public Economics 90(4), 631-656.

Bonica, A. (2013). Ideology and interests in the political marketplace. American Journal of Political Science $57(2), 294-311$.

Bonica, A. (2014). Mapping the ideological marketplace. American Journal of Political Science 58(2), $367-386$.

Bonica, A. (2017). Professional networks, early fundraising, and electoral success. Election Law Journal 16(1), 153-171.

Brunell, T. L. (2005). The relationship between political parties and interest groups: Explaining patterns of pac contributions to candidates for congress. Political Research Quarterly 58(4), 681-688.

Calvert, R. L. (1985). Robustness of the multidimensional voting model: Candidate motivations, uncertainty, and convergence. American Journal of Political Science, 69-95.

Cotton, C. (2009). Should we tax or cap political contributions? a lobbying model with policy favors and access. Journal of Public Economics 93(7), 831-842.

Cotton, C. (2012). Pay-to-play politics: Informational lobbying and contribution limits when money buys access. Journal of Public Economics 96(3), 369-386.

Crosson, J. M., A. C. Furnas, and G. M. Lorenz (2020). Polarized pluralism organizational preferences and biases in the american pressure system. American Journal of Political Science. 
Dahm, M. and N. Porteiro (2008). Side effects of campaign finance reform. Journal of the European Economic Association 6(5), 1057-1077.

de Figueiredo, J. M. and C. M. Cameron (2019). Endogenous cost lobbying: Theory and evidence. Working Paper.

Dimmery, D. and A. Peterson (2016). Shining the light on dark money: Political spending by nonprofits. RSF: The Russell Sage Foundation Journal of the Social Sciences 2(7), 51-68.

Duggan, J. (2008). Candidate Objectives and Electoral Equilibrium. In B. Weingast and W. Donald (Eds.), The Oxford Handbook of Political Economy, Chapter 4, pp. 21. Oxford: Oxford University Press.

Duggan, J. and J. Gao (2019). Lobbying as a multidimensional tug of war. Social Choice and Welfare, 1-26.

Felli, L. and A. Merlo (2007). If you cannot get your friends elected, lobby your enemies. Journal of the European Economic Association 5(2-3), 624-635.

Fouirnaies, A. (2020). How do campaign spending limits affect elections? evidence from the united kingdom 1885-2019. American Political Science Review, 1-17.

Fouirnaies, A. and A. Fowler (2021). Do campaign contributions buy favorable policies? evidence from the insurance industry. Political Science Research and Methods, 1-15.

Fouirnaies, A. and A. B. Hall (2018). How do interest groups seek access to committees? American Journal of Political Science 62(1), 132-147.

Fowler, A., H. Garro, and J. L. Spenkuch (2020). Quid pro quo? corporate returns to campaign contributions. The Journal of Politics 82(3), 000-000.

Gerber, A. (1998). Estimating the effect of campaign spending on senate election outcomes using instrumental variables. American Political science review, 401-411.

Groll, T. and C. J. Ellis (2017). Repeated lobbying by commercial lobbyists and special interests. Economic Inquiry 55(4), 1868-1897.

Groseclose, T. (2001). A model of candidate location when one candidate has a valence advantage. American Journal of Political Science, 862-886.

Grossman, G. M. and E. Helpman (2001). Special interest politics. MIT press.

Grumbach, J. and P. Pierson (2019). Are large corporations politically moderate? using money in politics to infer the preferences of business. Working Paper. 
Hall, R. L. and A. V. Deardorff (2006). Lobbying as legislative subsidy. American Political Science Review 100(1), 69-84.

Hirsch, A. V., K. Kang, B. P. Montagnes, and H. Y. You (2020). Lobbyists as gatekeepers: Theory and evidence. Working Paper.

Judd, G. (2019). Access and lobbying in legislatures. Working Paper.

Kalla, J. L. and D. E. Broockman (2016). Campaign contributions facilitate access to congressional officials: A randomized field experiment. American Journal of Political Science 60(3), 545-558.

Kroeger, M. (2020). Groups as lawmakers: Group-sponsored bills in the california state legislature. State Politics and Policy Quarterly (forthcoming).

Lake, J. (2015). Revisiting the link between pac contributions and lobbying expenditures. European Journal of Political Economy 37, 86-101.

Lorenz, G. M., A. C. Furnas, and J. M. Crosson (2020). Large-n bill positions data from maplight.org: What can we learn from interest groups' publicly observable legislative positions? Interest Groups $\mathscr{E}^{3}$ Advocacy, $1-19$.

McKay, A. (2010). The effects of interest groups' ideology on their pac and lobbying expenditures. Business and Politics 12(2), 1-21.

McKay, A. (2018a). What do campaign contributions buy? lobbyists' strategic giving. Interest Groups and Advocacy 7(1), 1-18.

McKay, A. M. (2018b). Fundraising for favors? linking lobbyist-hosted fundraisers to legislative benefits. Political Research Quarterly 71(4), 869-880.

Meirowitz, A. (2008). Electoral contests, incumbency advantages, and campaign finance. The Journal of Politics 70(3), 681-699.

Miller, K. M. (2019). The divided labor of attack advertising in congressional campaigns. The Journal of Politics 81(3), 805-819.

Montagnes, P. and R. Van Weelden (2019). On the incentives to exacerbate polarization. Working Paper.

Morton, R. B. and R. B. Myerson (2012). Decisiveness of contributors' perceptions in elections. Economic Theory $49(3), 571-590$. 
Powell, E. N. and J. Grimmer (2016). Money in exile: Campaign contributions and committee access. The Journal of Politics 78(4), 974-988.

Schnakenberg, K. E. and I. R. Turner (2021). Helping friends or influencing foes: Electoral and policy effects of campaign finance contributions. American Journal of Political Science 65(1), 88-100.

Sprick Schuster, S. (2020). Does campaign spending affect election outcomes? new evidence from transactionlevel disbursement data. The Journal of Politics.

Strickland, J. (2019). A paradox of political reform: Shadow interests in the us states. American Politics Research 47(4), 887-914.

Thieme, S. (2020). Moderation or strategy? political giving by corporations and trade groups. Journal of Politics (forthcoming).

Titl, V. and B. Geys (2019). Political donations and the allocation of public procurement contracts. European Economic Review 111, 443-458.

Tripathi, M., S. Ansolabehere, and J. M. Snyder (2002). Are pac contributions and lobbying linked? new evidence from the 1995 lobby disclosure act. Business and politics 4(2), 131-155.

Wolton, S. (n.p.). Lobbying, inside and out: How special interest groups influence policy choices. Quarterly Journal of Political Science.

You, H. Y. (2020). Dynamic lobbying: Evidence from foreign lobbying in the us congress. Working Paper. 


\section{A Proofs}

Proof of Proposition 1. It is enough to consider $v_{L}^{*}=\frac{\pi+\left(2-a_{L}\right) \epsilon_{L}+a_{R} \epsilon_{R}}{2}$ (with similar arguments holding for $\left.v_{R}^{*}\right)$. Valence produced by $G_{L}$ is strictly increasing in polarization $(\pi)$ and, since $\alpha_{L}, \alpha_{R} \in[0,1]$, is weakly increasing also in own relative extremism $\left(\epsilon_{L}\right)$, and opposing group extremism $\left(\epsilon_{R}\right)$.

The derivative of $v_{L}^{*}$ with respect to concessions granted by its aligned politician $\left(\alpha_{L}\right)$, is positive when $\epsilon_{L}<0$ (i.e., the group is more moderate than the politician) and negative when $\epsilon_{L}>0$.

The derivative of $v_{L}^{*}$ with respect to concessions granted by its opposing politician $\left(\alpha_{R}\right)$ to the opposing group, is positive when $\epsilon_{R}>0$ (i.e., the group is more extreme than the politician) and negative when $\epsilon_{R}<0$.

Proof of Proposition 2. The arguments in text establish that a candidate granting any concessions to an aligned interest group that is relatively more extreme is strictly dominated, so we may conclude $a_{L}^{*}=0$ when $\epsilon_{L}>0$ and $a_{R}^{*}=0$ when $\epsilon_{R}>0$. One implication of these results is to set an upper bound on the value of $a_{L} \epsilon_{L}$ and $a_{R} \epsilon_{R}$ of 0 . In turn, this sets a weakly higher lower bound for $\pi$, viz., $\pi \geq 1-a_{L} \epsilon_{L} \geq 1$.

What remains to be shown is that when $\epsilon_{L}<0$ (resp., $\epsilon_{R}<0$ ), $a_{L}^{*}=1$ (resp., $a_{R}^{*}=1$ ). In making explicit the candidates' expected utilities, we may plug in $v_{L}^{*}-v_{R}^{*}=\left(1-a_{L}\right) \epsilon_{L}-\left(1-a_{R}\right) \epsilon_{R}$. The expected utility for the left politician is given by: ${ }^{13}$

$$
\begin{aligned}
u_{P_{L}}\left(a_{L}\right) & =-\operatorname{Pr}\left(P_{L} \text { wins } \mid a_{L}\right)\left(\tilde{x}_{L}-\hat{x}_{P_{L}}\right)^{2}-\left[1-\operatorname{Pr}\left(P_{L} \text { wins } \mid a_{L}\right)\right]\left(\tilde{x}_{R}-\hat{x}_{P_{L}}\right)^{2} \\
& =\left(1+2 \mu+a_{R} \epsilon_{R}-a_{L} \epsilon_{L}+\frac{\left(1-a_{L}\right) \epsilon_{L}-\left(1-a_{R}\right) \epsilon_{R}}{\pi+a_{R} \epsilon_{R}+a_{L} \epsilon_{L}}\right)\left[\left(\pi+a_{R} \epsilon_{R}\right)^{2}-\left(a_{L} \epsilon_{L}\right)^{2}\right] / 2-\left(\pi+a_{R} \epsilon_{R}\right)^{2} / 2
\end{aligned}
$$

The expected utility for the right politician is given by: ${ }^{14}$

$$
\begin{aligned}
u_{P_{R}}\left(a_{R}\right) & =-\operatorname{Pr}\left(P_{R} \text { wins } \mid a_{R}\right)\left(\tilde{x}_{R}-\hat{x}_{P_{R}}\right)^{2}-\left[1-\operatorname{Pr}\left(P_{R} \text { wins } \mid a_{R}\right)\right]\left(\tilde{x}_{L}-\hat{x}_{P_{R}}\right)^{2} \\
& =\left(1-2 \mu+a_{L} \epsilon_{L}-a_{R} \epsilon_{R}+\frac{\left(1-a_{R}\right) \epsilon_{R}-\left(1-a_{L}\right) \epsilon_{L}}{\pi+a_{R} \epsilon_{R}+a_{L} \epsilon_{L}}\right)\left[\left(\pi+a_{L} \epsilon_{L}\right)^{2}-\left(a_{R} \epsilon_{R}\right)^{2}\right] / 2-\left(\pi+a_{L} \epsilon_{L}\right)^{2} / 2 .
\end{aligned}
$$

We consider, without loss of generality, $P_{L}$ 's concessions decision henceforth. We seek to demonstrate that expected utility is increasing for all $a_{L} \in[0,1]$ when $\epsilon_{L}<0$. We may then conclude that the politician optimally provides full concessions, i.e., $a_{L}^{*}=1$ when the interest group is more moderate.

Consider the derivative of $P_{L}$ 's expected utility with respect to the candidate's choice variable, $a_{L}$, with

\footnotetext{
${ }^{13}$ Note that $-\left(\tilde{x}_{L}-\hat{x}_{P_{L}}\right)^{2}=-\left(-a_{L} \epsilon_{L}\right)^{2}=-\left(a_{L} \epsilon_{L}\right)^{2}$ and that $-\left(\tilde{x}_{R}-\hat{x}_{P_{L}}\right)^{2}=-\left(\hat{x}_{P_{R}}+a_{R} \epsilon_{R}-\hat{x}_{P_{L}}\right)^{2}=-\left(\pi+a_{R} \epsilon_{R}\right)^{2}$.

${ }^{14}$ Note that $-\left(\tilde{x}_{R}-\hat{x}_{P_{R}}\right)^{2}=-\left(a_{R} \epsilon_{R}\right)^{2}$ and that $-\left(\tilde{x}_{L}-\hat{x}_{P_{R}}\right)^{2}=-\left(\hat{x}_{P_{L}}-a_{L} \epsilon_{L}-\hat{x}_{P_{R}}\right)^{2}=-\left(-\pi-a_{L} \epsilon_{L}\right)^{2}=-\left(\pi+a_{L} \epsilon_{L}\right)^{2}$.
} 
some helpful rearrangement and simplification

$$
\begin{aligned}
& \frac{\partial}{\partial a_{L}}\left(\left(1+2 \mu+a_{R} \epsilon_{R}-a_{L} \epsilon_{L}+\frac{\left(1-a_{L}\right) \epsilon_{L}-\left(1-a_{R}\right) \epsilon_{R}}{\pi+a_{R} \epsilon_{R}+a_{L} \epsilon_{L}}\right)\left[\left(\pi+a_{R} \epsilon_{R}\right)^{2}-\left(a_{L} \epsilon_{L}\right)^{2}\right] / 2-\left(\pi+a_{R} \epsilon_{R}\right)^{2} / 2\right) \\
= & \frac{\partial}{\partial a_{L}}\left(1+2 \mu+a_{R} \epsilon_{R}-a_{L} \epsilon_{L}\right)\left[\left(\pi+a_{R} \epsilon_{R}\right)^{2}-\left(a_{L} \epsilon_{L}\right)^{2}\right] \\
& +\frac{\partial}{\partial a_{L}}\left[\left(1-a_{L}\right) \epsilon_{L}-\left(1-a_{R}\right) \epsilon_{R}\right]\left(\pi+a_{R} \epsilon_{R}-a_{L} \epsilon_{L}\right) \\
= & -\epsilon_{L}\left(\left(\pi+a_{R} \epsilon_{R}\right)^{2}-\left(a_{L} \epsilon_{L}\right)^{2}+2 a_{L} \epsilon_{L}\left(1+2 \mu+a_{R} \epsilon_{R}-a_{L} \epsilon_{L}\right)\right) \\
& -\epsilon_{L}\left(\pi+a_{R} \epsilon_{R}-a_{L} \epsilon_{L}+\left[\left(1-a_{L}\right) \epsilon_{L}-\left(1-a_{R}\right) \epsilon_{R}\right]\right) .
\end{aligned}
$$

To reiterate, we seek to demonstrate that the sum of (13) and (14) are positive when $\epsilon_{L}<0$ for all $a_{L} \in[0,1]$. As such, we may drop the $-2 \epsilon_{L}>0$ coefficient. Further, it is helpful to split the expression across (13) and (14) into three components:

$$
\begin{aligned}
& \left(\pi+a_{R} \epsilon_{R}\right)^{2}-\left(a_{L} \epsilon_{L}\right)^{2} \\
& 2 a_{L} \epsilon_{L}\left(1+2 \mu+a_{R} \epsilon_{R}-a_{L} \epsilon_{L}\right) \\
& \pi+\left(1-2 a_{L}\right) \epsilon_{L}-\left(1-2 a_{R}\right) \epsilon_{R} .
\end{aligned}
$$

Recalling $\pi+a_{R} \epsilon_{R} \geq 1-a_{L} \epsilon_{L}$, the expression in (15) must be greater than or equal to $1-2 a_{L} \epsilon_{L}$.

Turning to (16), note first that $a_{L} \epsilon_{L} \leq 0$, so the entire expression is thus bounded below by $a_{L} \epsilon_{L}$ times the (rather, an) upper bound of $1+2 \mu+a_{R} \epsilon_{R}-a_{L} \epsilon_{L}$. Noting $2 \geq 1+2|\mu|$ and $a_{R} \epsilon_{R} \leq 0$, it follows that $1+2 \mu+a_{R} \epsilon_{R}-a_{L} \epsilon_{L} \leq 2-a_{L} \epsilon_{L}$. Hence, the expression in (16) is greater than or equal to $2 a_{L} \epsilon_{L}\left(2-a_{L} \epsilon_{L}\right)$.

Adding the lower bounds, the expressions in (15) and (16) are greater than or equal to $1+2 a \epsilon_{L}-2\left(a \epsilon_{L}\right)^{2}$. If the expression in (17) plus $1+2 a \epsilon_{L}-2\left(a \epsilon_{L}\right)^{2}$ is greater than 0 , we may conclude the candidate's expected utility is increasing over $a_{L} \in[0,1]$, and the optimal choice is $a_{L}=1$. Simplifying this quantity yields:

$$
\pi+\left(1-2 a_{L}\right) \epsilon_{L}-\left(1-2 a_{R}\right) \epsilon_{R}+1+2 a \epsilon_{L}-2\left(a \epsilon_{L}\right)^{2}=\pi+a_{R} \epsilon_{R}+\epsilon_{L}-\left(1-a_{R}\right) \epsilon_{R}+1-2\left(a_{L} \epsilon_{L}\right)^{2}
$$

Since $\pi+a_{R} \epsilon_{R} \geq 1-\epsilon_{L},-\left(1-a_{R}\right) \epsilon_{R} \geq-1$, and $1 \geq\left(a \epsilon_{L}\right)^{2}$, we have:

$$
\pi+a_{R} \epsilon_{R}+\epsilon_{L}-\left(1-a_{R}\right) \epsilon_{R}+1-2\left(a_{L} \epsilon_{L}\right)^{2} \geq 2-2\left(a_{L} \epsilon_{L}\right)^{2} \geq 0, \forall a_{L} \in[0,1]
$$

which was to be shown. 
Proof of Corollary 1. The distance between the final policies candidates would enact if elected is:

$$
\tilde{x}_{R}^{*}-\tilde{x}_{L}^{*}= \begin{cases}\pi+\epsilon_{L}+\epsilon_{R} & \text { if } \epsilon_{L}<0, \epsilon_{R}<0 \\ \pi+\epsilon_{L} & \text { if } \epsilon_{L}<0, \epsilon_{R}>0 \\ \pi+\epsilon_{R} & \text { if } \epsilon_{L}>0, \epsilon_{R}<0 \\ \pi & \text { if } \epsilon_{L}>0, \epsilon_{R}>0\end{cases}
$$

The difference between final policies is always increasing in $\pi$ and never decreasing in $\epsilon_{L}$ and $\epsilon_{R}$.

Proof of Corollary 2. Equilibrium spending by the two groups is:

$$
\left(s_{L}^{*}, s_{R}^{*}\right)= \begin{cases}\left(\left(\pi+\epsilon_{L}+\epsilon_{R}\right)^{2} / 8,\left(\pi+\epsilon_{L}+\epsilon_{R}\right)^{2} / 8\right) & \text { if } \epsilon_{L}<0, \epsilon_{R}<0 \\ \left(\left(\pi+\epsilon_{L}\right)^{2} / 8,\left(\pi+2 \epsilon_{R}+\epsilon_{L}\right)^{2} / 8\right) & \text { if } \epsilon_{L}<0, \epsilon_{R}>0 \\ \left(\left(\pi+2 \epsilon_{L}+\epsilon_{R}\right)^{2} / 8,\left(\pi+\epsilon_{R}\right)^{2} / 8\right) & \text { if } \epsilon_{L}>0, \epsilon_{R}<0 \\ \left(\left(\pi+2 \epsilon_{L}\right)^{2} / 8,\left(\pi+2 \epsilon_{R}\right)^{2} / 8\right) & \text { if } \epsilon_{L}>0, \epsilon_{R}>0 .\end{cases}
$$

Noting that $\pi+2 \epsilon_{L} \geq 0, \pi+2 \epsilon_{R} \geq 0, \pi+2 \epsilon_{R}+\epsilon_{L} \geq 0$, and $\pi+\epsilon_{R}+2 \epsilon_{L} \geq 0$, it is straightforward to establish the following relationships:

Table 1: Comparative statics of each group's and total spending $\mathrm{w} / \mathrm{r} / \mathrm{t} \epsilon_{L}, \epsilon_{R}, \pi$, for all cases

\begin{tabular}{c|c|c|c} 
& $\partial s_{L}^{*} / \partial \epsilon_{L}$ & $\partial s_{L}^{*} / \partial \epsilon_{R}$ & $\partial s_{L}^{*} / \partial \pi$ \\
\hline \hline$\epsilon_{L}<0, \epsilon_{R}<0$ & + & + & + \\
$\epsilon_{L}<0, \epsilon_{R}>0$ & + & 0 & + \\
$\epsilon_{L}>0, \epsilon_{R}<0$ & + & + & + \\
$\epsilon_{L}>0, \epsilon_{R}>0$ & + & 0 & + \\
\hline \hline$\epsilon_{L}<0, \epsilon_{R}<0$ & $\partial s_{R}^{*} / \partial \epsilon_{L}$ & $\partial s_{R}^{*} / \partial \epsilon_{R}$ & $\partial s_{R}^{*} / \partial \pi$ \\
$\epsilon_{L}<0, \epsilon_{R}>0$ & + & + & + \\
$\epsilon_{L}>0, \epsilon_{R}<0$ & + & + & + \\
$\epsilon_{L}>0, \epsilon_{R}>0$ & 0 & + & + \\
\end{tabular}


Proof of Corollary 3. The equilibrium probability that $P_{L}$ wins is:

$$
\operatorname{Pr}\left(P_{L} \text { wins }\right)= \begin{cases}\left(1+2 \mu+\epsilon_{R}-\epsilon_{L}\right) / 2 & \text { if } \epsilon_{L}<0, \epsilon_{R}<0 \\ \left(1+2 \mu-\epsilon_{L}-\frac{\epsilon_{R}}{\pi+\epsilon_{L}}\right) / 2 & \text { if } \epsilon_{L}<0, \epsilon_{R}>0 \\ \left(1+2 \mu+\epsilon_{R}+\frac{\epsilon_{L}}{\pi+\epsilon_{R}}\right) / 2 & \text { if } \epsilon_{L}>0, \epsilon_{R}<0 \\ \left(1+2 \mu+\frac{\epsilon_{L}-\epsilon_{R}}{\pi}\right) / 2 & \text { if } \epsilon_{L}>0, \epsilon_{R}>0\end{cases}
$$

Table 2: Comparative statics of probability left group wins $\mathrm{w} / \mathrm{r} / \mathrm{t} \epsilon_{L}, \epsilon_{R}, \pi$, for all cases

\begin{tabular}{|c|c|c|c|}
\hline & $\partial \operatorname{Pr}(L$ wins $) / \partial \epsilon_{L}$ & $\partial \operatorname{Pr}(L$ wins $) / \partial \epsilon_{R}$ & $\partial \operatorname{Pr}(L$ wins $) / \partial \pi$ \\
\hline$\epsilon_{L}<0, \epsilon_{R}<0$ & $-1<0$ & $1>0$ & $0=0$ \\
\hline$\epsilon_{L}<0, \epsilon_{R}>0$ & $-1+\frac{\epsilon_{R}}{\left(\pi+\epsilon_{L}\right)^{2}}<0$ & $-\frac{1}{\pi+\epsilon_{L}}<0$ & $\frac{\epsilon_{R}}{\left(\pi+\epsilon_{L}\right)^{2}}>0$ \\
\hline$\epsilon_{L}>0, \epsilon_{R}<0$ & $\frac{1}{\pi+\epsilon_{R}}>0$ & $1-\frac{\epsilon_{L}}{\left(\pi+\epsilon_{R}\right)^{2}}>0$ & $-\frac{\epsilon_{L}}{\left(\pi+\epsilon_{R}\right)^{2}}<0$ \\
\hline$\epsilon_{L}>0, \epsilon_{R}>0$ & $\frac{1}{\pi}>0$ & $-\frac{1}{\pi}<0$ & $-\frac{\epsilon_{L}-\epsilon_{R}}{\pi^{2}} \gtrless 0$ \\
\hline
\end{tabular}

Proof of Propositions 3. Because a party choose a politician's ideal point, and not a level of polarization or alignment, we revert to the original ideal point paramterization for this proof. We show this for the right party; the left party's problem is similar.

Note that from the party's perspective, all politicians more extreme than the interest group are equivalent. They have the same final policy, the same spending, and the same probability of winning. Therefore we need only consider politicians (weakly) more moderate than the interest group and can assume $\alpha=0$. Therefore, the right party's maximization problem is

$$
\begin{aligned}
& \max _{\hat{x}_{P_{R}}}-\left(\hat{x}_{O_{R}}-\hat{x}_{P_{R}}\right)^{2}\left(\frac{1}{2}-\frac{\hat{x}_{P_{R}}+\hat{x}_{P_{L}}}{2}+\frac{\hat{x}_{G_{R}}+\hat{x}_{G_{L}}-\hat{x}_{P_{R}}-\hat{x}_{P_{L}}}{2\left(\hat{x}_{P_{R}}-\hat{x}_{P_{L}}\right)}\right) \\
&-\left(\hat{x}_{O_{R}}-\hat{x}_{P_{L}}\right)^{2}\left(\frac{1}{2}+\frac{\hat{x}_{P_{R}}+\hat{x}_{P_{L}}}{2}-\frac{\hat{x}_{G_{R}}+\hat{x}_{G_{L}}-\hat{x}_{P_{R}}-\hat{x}_{P_{L}}}{2\left(\hat{x}_{P_{R}}-\hat{x}_{P_{L}}\right)}\right) \\
& \frac{\partial E U_{O_{R}}\left(\hat{x}_{P_{R}}\right)}{\partial \hat{x}_{P_{R}}}=\frac{1}{2}\left(-\hat{x}_{G_{L}}-\hat{x}_{G_{R}}+4 \hat{x}_{O_{R}} \hat{x}_{P_{L}}-4 \hat{x}_{O_{R}} \hat{x}_{P_{R}}-\hat{x}_{P_{L}}^{2}-2 \hat{x}_{P_{L}} \hat{x}_{P_{R}}+2 \hat{x}_{P_{L}}+3 \hat{x}_{P_{R}}^{2}\right)
\end{aligned}
$$

Note that this derivative is strictly decreasing on the interval $\left[\frac{1}{2}, \hat{x}_{O_{R}}\right]$. Therefore it is maximized on this interval at $\frac{1}{2}$. Because we need only consider ideal points on the interval $\left[\frac{1}{2}, \hat{x}_{G_{R}}\right]$, this implies that when 
$\hat{x}_{O_{R}} \geq \hat{x}_{G_{R}}$ the optimal politician ideal point is $\frac{1}{2}$. It remains to be shown that when $\hat{x}_{O_{R}} \leq \hat{x}_{G_{R}}, \frac{1}{2}$ is better than any other ideal point in the $\left[\hat{x}_{O_{R}}, \hat{x}_{G_{R}}\right]$ interval.

We need

$$
\begin{aligned}
& -\left(\hat{x}_{O_{R}}-\frac{1}{2}\right)^{2}\left(\frac{1}{2}-\frac{\frac{1}{2}+\hat{x}_{P_{L}}}{2}+\frac{\hat{x}_{G_{R}}+\hat{x}_{G_{L}}-\frac{1}{2}-\hat{x}_{P_{L}}}{2\left(\frac{1}{2}-\hat{x}_{P_{L}}\right)}\right) \\
& -\left(\hat{x}_{O_{R}}-\hat{x}_{P_{L}}\right)^{2}\left(\frac{1}{2}+\frac{\frac{1}{2}+\hat{x}_{P_{L}}}{2}-\frac{\hat{x}_{G_{R}}+\hat{x}_{G_{L}}-\frac{1}{2}-\hat{x}_{P_{L}}}{2\left(\frac{1}{2}-\hat{x}_{P_{L}}\right)}\right) \\
& \geq-\left(\hat{x}_{O_{R}}-\hat{x}_{P_{R}}\right)^{2}\left(\frac{1}{2}-\frac{\hat{x}_{P_{R}}+\hat{x}_{P_{L}}}{2}+\frac{\hat{x}_{G_{R}}+\hat{x}_{G_{L}}-\hat{x}_{P_{R}}-\hat{x}_{P_{L}}}{2\left(\hat{x}_{P_{R}}-\hat{x}_{P_{L}}\right)}\right) \\
& -\left(\hat{x}_{O_{R}}-\hat{x}_{P_{L}}\right)^{2}\left(\frac{1}{2}+\frac{\hat{x}_{P_{R}}+\hat{x}_{P_{L}}}{2}-\frac{\hat{x}_{G_{R}}+\hat{x}_{G_{L}}-\hat{x}_{P_{R}}-\hat{x}_{P_{L}}}{2\left(\hat{x}_{P_{R}}-\hat{x}_{P_{L}}\right)}\right)
\end{aligned}
$$

which is always true when $\hat{x}_{P_{R}} \in\left[\hat{x}_{O_{R}}, \hat{x}_{G_{R}}\right]$.

Proof of Corollary 4. We show this proof for $O_{R} ; O_{L}$ is similar.

From Proposition 3 , we know that $\hat{x}_{P_{R}}^{*}=\frac{1}{2}$. The expected utility for the party when $\hat{x}_{P_{R}}^{*}=\frac{1}{2}$ is

$$
\begin{aligned}
& -\left(\hat{x}_{O_{R}}-\frac{1}{2}\right)^{2}\left(\frac{1}{2}-\frac{\frac{1}{2}+\hat{x}_{P_{L}}}{2}+\frac{\hat{x}_{G_{R}}+\hat{x}_{G_{L}}-\frac{1}{2}-\hat{x}_{P_{L}}}{2\left(\frac{1}{2}-\hat{x}_{P_{L}}\right)}\right) \\
& -\left(\hat{x}_{O_{R}}-\hat{x}_{P_{L}}\right)^{2}\left(\frac{1}{2}+\frac{\frac{1}{2}+\hat{x}_{P_{L}}}{2}-\frac{\hat{x}_{G_{R}}+\hat{x}_{G_{L}}-\frac{1}{2}-\hat{x}_{P_{L}}}{2\left(\frac{1}{2}-\hat{x}_{P_{L}}\right)}\right)
\end{aligned}
$$

Taking the derivative with respect to $\hat{x}_{G_{R}}$, we have

$$
\hat{x}_{O_{R}}-\frac{\hat{x}_{P_{L}}}{2}-\frac{1}{4}
$$

which is always greater than 0 . 